\title{
Robust pedestrian trajectory reconstruction from inertial sensor
}

\author{
Bertrand Beaufils*, Frédéric Chazal ${ }^{\dagger}$, Marc Grelet ${ }^{\ddagger}$ and Bertrand Michel ${ }^{\S}$ \\ $* \ddagger$ Sysnav, 57 Rue de Montigny, 27200 Vernon, France \\ $* \dagger \S$ Inria Saclay team DataShape, 1 Rue Honoré d'Estienne d'Orves, 91120 Palaiseau, France \\ $\S$ Centrale Nantes Informatic and Mathematics Department, 1 Rue de La Noe, 44300 Nantes, France
}

Email: *bertrand.beaufils@ sysnav.fr, ${ }^{\dagger}$ frederic.chazal@inria.fr, ${ }^{\dagger}$ marc.grelet@ sysnav.fr, ${ }^{\S}$ bertrand.michel@ec-nantes.fr

\begin{abstract}
In this paper, a strides detection algorithm combined with a technique inspired by Zero Velocity Update (ZUPT) is proposed using inertial sensors worn on the ankle. This innovative approach based on a sensors alignment and machine learning can detect both normal walking strides and atypical strides such as small steps, side steps and backward walking that existing methods struggle to detect. As a consequence, the trajectory reconstruction achieves better performances in daily life contexts for example, where a lot of these kinds of strides are performed in narrow areas such as in a house. It is also robust in critical situations, when for example the wearer is sitting and moving the ankle or bicycling, while most algorithms in the literature would wrongly detect strides and produce error in the trajectory reconstruction by generating movements.

Our algorithm is evaluated on more than 7800 strides from seven different subjects performing several activities. We validated the trajectory reconstruction during motion capture sessions by analyzing the stride length. Finally, we tested the algorithm in a challenging situation by plotting the computed trajectory on the building map of an 5 hours and 30 minutes office worker recording.
\end{abstract}

\section{INTRODUCTION}

The emergence of Global Navigation Satellite System (GNSS) receivers in the 2000s has changed the perception of navigation. While they are commonly used in outdoor environments they fail to produce accurate localization due to poor reception in many situations, for example in tunnels, indoor parking, in the forest, inside buildings etc. Instead, bodymounted inertial measurement units (IMUs) can be used to record the movements of pedestrians, providing an estimate of their motion relative to a known origin. Unlike infrastructuredependent localization systems such as map matching, Wi-Fi [1], Radio Frequency Identification [2] or ultra-wideband [3], body-mounted IMUs are lightweight and can be rapidly and easily deployed.

In this context, Sysnav has developed WATA systems (Wearable Ankle Trajectory Analyzer) based on magneto-inertial sensors [4], [5], to enable trajectory reconstruction. Here we consider an ankle worn device for dead reckoning. The strategy which consists in the integration of the linear acceleration and angular velocity data from the unit may rapidly cumulate large errors due to IMUs drifts. To overcome this issue. we use a technique inspired by Zero Velocity Update (ZUPT) [6][9], which is an effective method to limit the accumulation of errors. It consists in correcting the speed drift by estimating the speed of the ankle when the foot is on the ground during the walk and then integrates the data only between two ZUPTs. Several studies [10], [11], propose to detect pedestrian movements and classify activities (such as walking, stairs climbing, running...) from inertial data. These approaches do not work well outside a controlled environment [12]. Moreover these methods based on sliding windows do not allow to detect individual strides. A few methods of stance detection have been proposed in the literature by tuning thresholds to determine the start and the end of the strides [13]-[15]. Sysnav first developed a similar approach based on the swing detection and a combination of criteria on the inertial data to determine the ZUPT instant (accelerations close to one $\mathrm{g}$ and small values of the angular velocity). These methods show good results for classical gait but they tend to fail for atypical strides such as stairs and small steps. In order to give an illustration of these limits, we ask a wearer to climb the stairs and get in a small corridor where he performs small steps before going back. We plot in Figure 1 the computed trajectory by the Sysnav algorithm. It illustrates that during the atypical strides in the

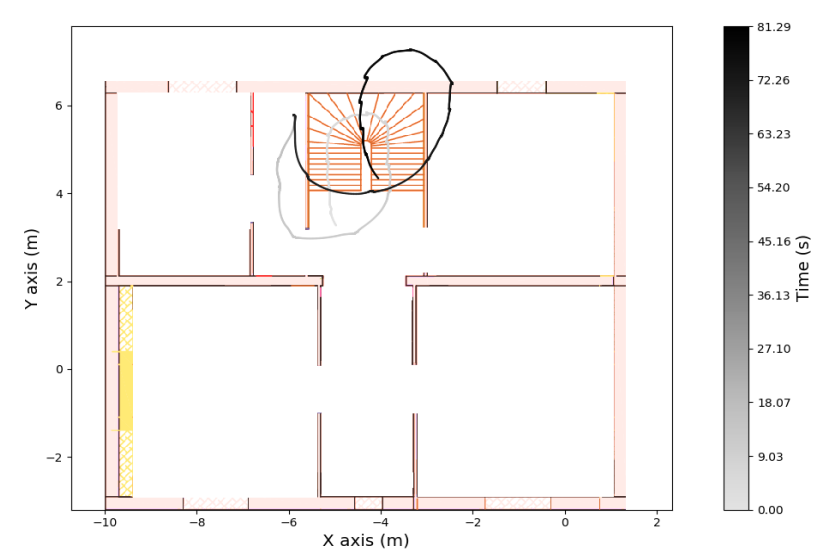

Fig. 1: Computed trajectory with a strides detection based on inertial thresholds. 
corridor, no stride is detected as the trajectory stays on the same point for dozens of seconds. In the end, the error is about two meters.

Other approaches use machine learning techniques on the frequency characteristics of the signals [16], [17]. These methods show good results when it is known that the pedestrian is walking but fail in a lot of real life situations. Indeed, several foot movements in sitting position and bicycling for example are wrongly detected as strides.

In this work we describe our step detector which is based on a innovative technique to compute a sensors alignment for inertial data that enables a extraction of intervals that may correspond to strides. The selection among them is performed by a classifier built with the Gradient Boosting Tree algorithm. The same approach can also be applied to recognize the activity of the performed step. Activity recognition can be a valuable information in many situations, for example in medical context, but we focus here on trajectory reconstruction.

\section{TERRESTRIAL REFERENCE FRAME COMPUTATION}

The system should be worn at the ankle as illustrated in Figure 2. In this default placement, the sensors record the inertial data in the reference frame defined by the $Z$ axis aligned with the leg and the $X$ axis aligned with the foot. However we observed that the device may be worn upside down and may turn around the ankle during the recording. The machine learning approach in this algorithm requires the 3-D inertial data to be in the same reference frame definition. In a previous work [18] we described a method that aligns the sensors based on geometric patterns of the angular velocity data. In this paper we present a more robust technique that has the particularity of removing the gravity from the acceleration data and compute a terrestrial reference frame. Indeed, the accelerometer in the device records the linear acceleration $(\Gamma)$ that is equal to the gravity added to the acceleration of the WATA system $\left(\Gamma^{\text {Ankle }}\right)$.

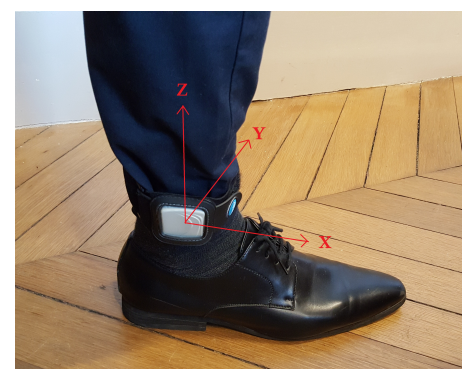

Fig. 2: Default device placement.

The main idea lies in the fact that in an inertial reference frame, the integration of $\Gamma^{\text {Ankle }}$ is equal to the difference of the ankle speed (a few meters per second for a pedestrian) that is small compared to the integration of the gravity. At any time $t$ in $\left[0, t_{\text {final }}\right]$, the device records the acceleration and angular velocity data (respectively $\Gamma_{B_{t}}(t)$ and $\Omega_{B_{t}}(t)$ in $\mathbb{R}^{3}$ ) in the body reference frame of the system $B_{t}$. With a no integration error of the angular velocity, $R_{t}$ solution of Equation 1 the following would be the rotation matrix between $B_{t}$ and $B_{0}$.

$$
\frac{d R_{t}}{d t}=-R_{t} \operatorname{Skew}\left(\Omega_{B_{t}}(t)\right)
$$

with $R_{0}=I_{3}$ and the Skew operator defined for all vector $n$ in $\mathbb{R}^{3}, n=\left(n_{x}, n_{y}, n_{z}\right)^{T}$ :

$$
\operatorname{Skew}(n)=\left(\begin{array}{ccc}
0 & -n_{z} & n_{y} \\
n_{z} & 0 & -n_{x} \\
-n_{y} & n_{x} & 0
\end{array}\right) .
$$

In practice, due to gyrometer imperfections (bias, non linearities, noise...), the product of the computed rotation matrix $R_{t}$ and $B_{t}$ only approximates $B_{0}$. We note $\hat{B}_{0}^{t}$ the resulting reference frame. We can now express for all $t$ the inertial data in the reference frame $\hat{B}_{0}^{t}$ as follows:

$$
\left\{\begin{array}{l}
\Gamma_{\hat{B}_{0}^{t}}(t)=R_{t} \Gamma_{B_{t}}(t) \\
\Omega_{\hat{B}_{0}^{t}}(t)=R_{t} \Omega_{B_{t}}(t)
\end{array}\right.
$$

Let $\Gamma_{B_{t}}^{\text {Ankle }}(t)$ be the acceleration of the WATA system without the gravity $g_{B_{t}}: \Gamma_{B_{t}}(t)=\Gamma_{B_{t}}^{\text {Ankle }}(t)+g_{B_{t}}$. Then the mean of the recorded acceleration projected in $\hat{B}_{0}^{t}$, on an interval $\Delta T$, is given by:

$$
\frac{1}{\Delta T} \int_{t}^{t+\Delta T} \Gamma_{\hat{B}_{0}^{u}}(u) d u=\frac{1}{\Delta T} \int_{t}^{t+\Delta T}\left(\Gamma_{\hat{B}_{0}^{u}}^{A n k l e}(u)+g_{\hat{B}_{0}^{u}}\right) d u .
$$

We assume that for a $\Delta T$ small enough, the reference frame $\hat{B}_{0}^{u}$ is constant for all $u$ in $[t, t+\Delta T]: \forall u \in[t, t+\Delta T], \hat{B}_{0}^{u}=$ $\hat{B}_{0}^{t}$. Namely, we consider that during a small period, the integration of the angular velocity produces no error. As a result, $g_{\hat{B}_{0}^{u}}$ is a constant $g_{\hat{B}_{0}^{t}}$ on this interval, we have:

$$
\begin{aligned}
\frac{1}{\Delta T} \int_{t}^{t+\Delta T} \Gamma_{\hat{B}_{0}^{u}}(u) d u & =\frac{1}{\Delta T} \int_{t}^{t+\Delta T} \Gamma_{\hat{B}_{0}^{t}}(u) d u \\
& =\frac{1}{\Delta T} \int_{t}^{t+\Delta T}\left(\Gamma_{\hat{B}_{0}^{t}}^{A n k l e}(u)+g_{\hat{B}_{0}^{t}}\right) d u \\
& =\frac{1}{\Delta T} \int_{t}^{t+\Delta T} \Gamma_{\hat{B}_{0}^{t}}^{A n k l e}(u) d u+g_{\hat{B}_{0}^{t}}
\end{aligned}
$$

Let $V_{\hat{B}_{0}^{t}}^{\text {Ankle }}(u)$ be the speed of the ankle in the reference frame $\hat{B}_{0 .}^{t}$ for all $u$ in $[t, t+\Delta T]$. From the equation above we can write:

$\frac{1}{\Delta T} \int_{t}^{t+\Delta T} \Gamma_{\hat{B}_{0}^{t}}(u) d u=\frac{V_{\hat{B}_{0}^{t}}^{\text {Ankle }}(t+\Delta T)-V_{\hat{B}_{0}^{t}}^{\text {Ankle }}(t)}{\Delta T}+g_{B_{0}}$.

For a sufficiently long duration of integration $\Delta T$, we assume that the speed difference of the ankle, between $t+\Delta T$ and $t$, divided by $\Delta T$ is small compared to the gravity:

$$
\frac{V_{\hat{B}_{0}^{t}}^{A n k l e}(t+\Delta T)-V_{\hat{B}_{0}^{t}}^{\text {Ankle }}(t)}{\Delta T} \ll g_{\hat{B}_{0}^{t}} .
$$

Thus, we can deduce the following equation:

$$
\frac{1}{\Delta T} \int_{t}^{t+\Delta T} \Gamma_{\hat{B}_{0}^{u}}(u) d u \approx g_{\hat{B}_{0}^{t}} .
$$

The assumption in Equation 3 is valid for large $\Delta T$ value. However, this approach requires to compute the mean of the acceleration in an inertial reference frame. Due to the integration drift with time, if $\Delta T$ is too large we have no guarantee that $\hat{B}_{0}^{u}$ equals $\hat{B}_{0}^{t}$ for all $u$ in $[t+\Delta T]$. In practice, we found a compromise by setting $\Delta T=15 \mathrm{~s}$.

Thanks to Equation 4 we can identify the gravity in the body reference frame at $t=0: g_{\hat{B}_{0}^{0}}=g_{B_{0}}$. If the angular velocity integration did not produce any error, for all $t>0 g_{\hat{B}_{0}^{t}}$ would be equals to $g_{B_{0}}$. In practice we observe that $g_{\hat{B}_{0}^{t}}$ changes with time. This variation is due to the integration drift. For 
all $t>0$ we can correct it by computing the rotation matrix $R_{t}^{g}$ that aligns $g_{\hat{B}_{0}^{t}}$ over time. We introduce the vector $a$ as follows:

$$
a=\lim _{d t \rightarrow 0} \frac{g_{\hat{B}_{0}^{t}} /\left\|g_{\hat{B}_{0}^{t}}\right\| \times g_{\hat{B}_{0}^{t+d t}} /\left\|g_{\hat{B}_{0}^{t+d t}}\right\|}{d t} .
$$

Then the rotation matrix $R_{t}^{g}$ is the solution of the following equation:

$$
\frac{d R_{t}^{g}}{d t}=-R_{t}^{g} \operatorname{Skew}(a)
$$

Then we can project the inertial data in the initial body reference frame for all $t>0$ :

$$
\begin{cases}\Gamma_{B_{0}}(t)= & R_{t}^{g} \Gamma_{\hat{B}_{0}^{t}}(t)=R_{t}^{g} R_{t} \Gamma_{B_{t}}(t), \\ \Omega_{B_{0}}(t)= & R_{t}^{g} \Omega_{\hat{B}_{0}^{t}}(t)=R_{t}^{g} R_{t} \Omega_{B_{t}}(t) .\end{cases}
$$

We now define a terrestrial reference frame $B^{\text {terr }}$ by considering the vector $-\frac{g_{B_{0}}}{\left\|g_{B_{0}}\right\|}$ as the new $Z^{\text {terr }}$ axis and choosing arbitrarily $X^{\text {terr }}$ and $Y^{\text {terr }}$ axes in order to build an orthonormal basis. We note $R^{\text {terr }}$ the rotation matrix between $B^{\text {terr }}$ and $B_{0}$. The inertial data projected in the terrestrial reference frame are given by the following for all $t$ :

$$
\left\{\begin{array}{l}
\Gamma_{B^{t e r r}}(t)=R^{\text {terr }} \Gamma_{B_{0}}(t), \\
\Omega_{B^{\text {terr }}}(t)=R^{\text {terr }} \Omega_{B_{0}}(t) .
\end{array}\right.
$$

We have now access to the acceleration of the ankle for all $t$ by removing the gravity $(\approx 9,81 \mathrm{~m} / \mathrm{s})$ from the $Z^{\text {terr }}$ axis:

$$
\Gamma_{B^{\text {terr }}}^{\text {Ankle }}(t)=\Gamma_{B^{\text {terr }}}(t)-\left(\begin{array}{c}
0 \\
0 \\
9,81
\end{array}\right) .
$$

The advantage of the attitude filter is the efficiency of its computation. This characteristic is necessary as we use $\Gamma_{B^{t e r r}}^{A n k l e}$ to compute a pseudo-speed that is one of the main features in our step detector. Indeed, due to the complexity of our application framework, it is difficult to describe a stride detector with only inertial models. In Section III, the computation of a pseudo-speed is introduced. It allows to extract a family of candidate intervals that may correspond to strides.

\section{PSEUDO-SPEED COMPUTATION}

\section{A. Integration of the ankle acceleration}

In previous Section II, we described the projection of the inertial data recorded by the device into a terrestrial reference frame $B^{t e r r}$. In this procedure, the gravity is removed from the acceleration that can be integrated to compute the pseudospeed of the ankle during the recording with an unknown initial condition. The first step of our algorithm is to detect phases of inactivity where we assume the ankle velocity as null. Let $\left\{\left(t_{1}^{0}, t_{1}^{1}\right), \ldots,\left(t_{i}^{0}, t_{i}^{1}\right), \ldots,\left(t_{n}^{0}, t_{n}^{1}\right)\right\}$ the $n$ detected couples of inactivity instants with the ankle in motion in between. We can integrate $\Gamma_{B^{t e r r}}^{A n k l e}$ between $t_{i}^{0}$ and $t_{i}^{1}$ chronologically and in the reverse time direction to compute what we call respectively forward speed $\left(V^{f o r}\right)$ and backward speed $\left(V^{b a c k}\right)$. We introduce here their general expression between two instants $a$ and $b$ with $a<b$ :

$$
\left\{\begin{array}{c}
V_{a, b}^{f o r}(t)=\int_{0}^{t-a} \Gamma_{B^{\text {terr }}}^{\text {Ankle }}(a+u) d u+V_{a, b}^{f o r}(a), \\
V_{a, b}^{\text {back }}(t)=\int_{0}^{b-t} \Gamma_{B^{\text {terr }}}^{\text {Ankle }}(b-u) d u+V_{a, b}^{\text {back }}(b) .
\end{array}\right.
$$

In particular, the instants $t_{i}^{0}$ and $t_{i}^{1}$ are defined as moments where the ankle is motionless so we assume $V_{i}^{f o r}\left(t_{i}^{0}\right)=0$ and $V_{i}^{\text {back }}\left(t_{i}^{1}\right)=0$. As a result we have:

$$
\begin{cases}V_{t_{i}^{0}, t_{i}^{1}}^{\text {for }}(t)= & \int_{0}^{t-t_{i}^{0}} \Gamma_{B^{t e r r}}^{\text {Ankle }}\left(t_{i}^{0}+u\right) d u, \\ V_{t_{i}^{0}, t_{i}^{1}}^{\text {back }}(t)= & \int_{0}^{t_{i}^{1}-t} \Gamma_{B^{t e r r}}^{\text {Ankle }}\left(t_{i}^{1}-u\right) d u .\end{cases}
$$

Since the integration drift cumulates errors with time, we make the assumption that for all $t$ in $[a, b]$, the more $t$ is close to $b$ the more $V_{a, b}^{f}$ produces errors and on the opposite the more $t$ is close to $a$ the more $V_{a, b}^{b}$ produces errors. That is why we compute the pseudo-speed $V_{a, b}$ as weighted mean between $a$ and $b$ :

$$
V_{a, b}(t)=V_{a, b}^{f o r}(t) \frac{b-t}{b-a}+V_{a, b}^{b a c k}(t) \frac{t-a}{b-a} .
$$

We note $t_{0}$ the first index of inactivity detected and $t_{n+1}$ the last one. For all $t<t_{0}$ we can only compute the backward speed as we do not know the inital condition for $t=0$ :

$$
V_{0, t_{0}}^{b}(t)=\int_{0}^{t_{0}-t} \Gamma_{B^{\text {terr }}}^{\text {Ankle }}\left(t_{0}-u\right) d u .
$$

On the contrary for all $t>t_{n+1}$ we can only compute the forward speed between $t_{n+1}$ and $t_{\text {final }}$ because we do not have any information on the speed of the ankle at the end of the recording:

$$
V_{t_{n+1}, t_{\text {final }}}^{f}(t)=\int_{0}^{t-t_{n+1}} \Gamma_{B^{t e r r}}^{\text {Ankle }}\left(t_{n+1}+u\right) d u .
$$

We can now define the pseudo-speed $V$ during all the recording, namely for all $t$ in $\left[0, t_{\text {final }}\right]$ :

$$
V(t)= \begin{cases}V_{0, t_{0}}^{\text {back }}(t) & \text { if } t<t_{0}, \\ V_{t_{i}^{0}, t_{i}^{1}}(t) & \text { if } t_{i}^{0}<t<t_{i}^{1}, \quad \forall i \in \llbracket 1, n \rrbracket, \\ V_{t_{n+1}, t_{\text {final }}}^{\text {for }}(t) & \text { if } t_{n+1}<t, \\ 0 & \text { otherwise. }\end{cases}
$$

\section{B. Pseudo-speed visualization}

A group of people of various ages and heights, have practiced several activities (walking, running, stairs, side steps, small steps...) wearing the WATA system with infrared markers during motion capture (MOCAP) sessions under video control. The cameras record the position of the device with high precision in a terrestrial reference frame defined at the beginning of the motion trial. Regarding the minimum of altitude and the video, we can detect when the foot is on the ground in the recording. Figure 3 illustrates a pattern in the inertial data that can be used to detect the beginning and the end of the strides during a walking phase. The contact of the foot with the ground is visible with a peak in the acceleration and a combination of conditions on the acceleration (one direction is close to one $\mathrm{g}$, the two others are close to zero) and angular velocity (local minima, swing phase identification etc.) seems indeed to be sufficient criteria and presented good results in [18]. Nevertheless this method has shown its limits in situations of atypical movements such as fast sides stepping and rapid descent of stairs. Figure 4 exhibits the problem as it is hard to detect when a stride occurs from the inertial data. 

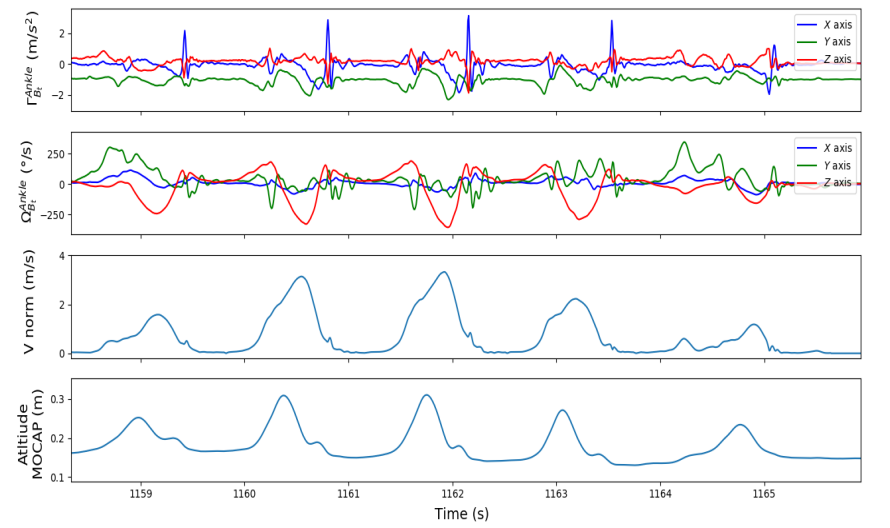

Fig. 3: Pseudo-speed norm during walking.
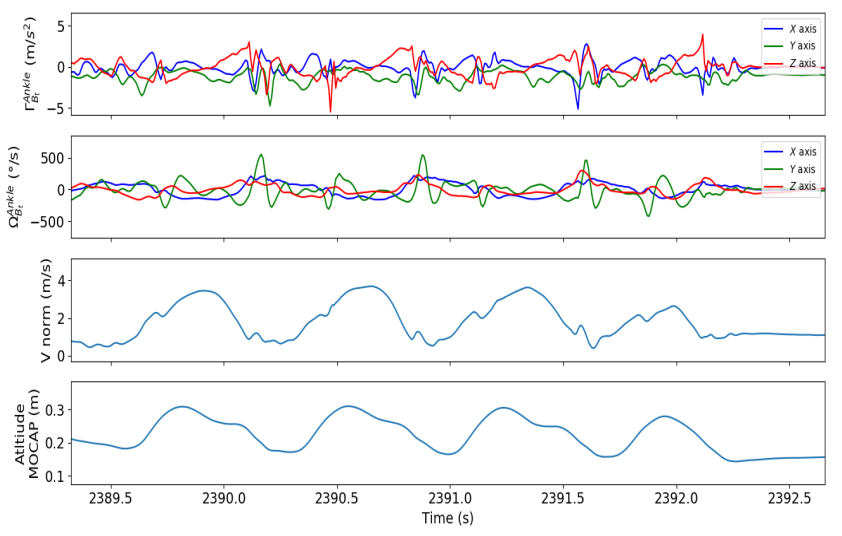

Fig. 4: Pseudo-speed norm during fast side stepping.

On the contrary we can see in both Figure 3 and Figure 4 that the norm of $V$ (Equation 14) is a good feature to solve this issue. The beginning and the end of a stride are defined by local minima around a maximum. However by following this procedure based on $V$ norm criteria, many intervals are wrongly extracted when the wearer is moving its ankle but not walking. The goal is now to select among these intervals which ones are true strides. We adopt a statistical learning approach to answer this problem.

\section{Algorithm}

\section{A. Preprocessing and database}

The first step of the algorithm is to project the inertial data in the terrestrial frame presented in Section II. Then we compute the pseudo-speed $V$ described in Section III and defined in Equation 14. We saw in previous Section III that a combination of criteria on the norm of the computed speed allows to detect the start and the end of the stride. We set wide threshold values in order to detect all types of strides (small steps, running, stairs etc.). However many intervals are wrongly selected when the wearer is moving its ankle during daily activities other than walking (bicycling, sitting in a car etc.) and when the WATA system is manipulated before being worn on the ankle. Indeed, the sensors start recording when the device is taken from its case and it can be carried with movement (in a backpack or pocket etc.) during an unknown amount of time. The goal is now to keep among these intervals those that are true strides. To answer this problem, we adopt a statistical learning approach.

From the intervals extraction above, a learning set is built from recordings of a group of people of various ages and heights practicing several activities. A binary label is affected to each interval indicating if it is a stride or not. Our database contains about 5000 positive intervals and also about 5000 negative intervals. In this binary classification problem, we adopt a strategy of supervised machine learning algorithm.

\section{B. Features engineering process}

For all recordings, the inertial data and pseudo-speed are projected in the terrestrial frame $B^{\text {terr }}$ whose the $Z^{\text {terr }}$ axis is aligned with the gravity and the two other axes ( $X^{\text {terr }}$ and $Y^{\text {terr }}$ ) axes are set arbitrarily (Section II). To be robust to this orientation that is dependent to each recording, in the following we compute a new rotation matrix around the $Z^{\text {terr }}$ axis for all selected intervals.

Let the variable $j$ in $\llbracket 1, N \rrbracket$ denotes the index of each interval. These intervals are defined by one start and end that we note start $_{j}$ and $e n d_{j}$. We assume that during the beginning and the end of a stride, when the foot is flat on the floor, the ankle is in pure rotation. This mechanics is described in [19] and is illustrated in Figure 5. From this observation, if the

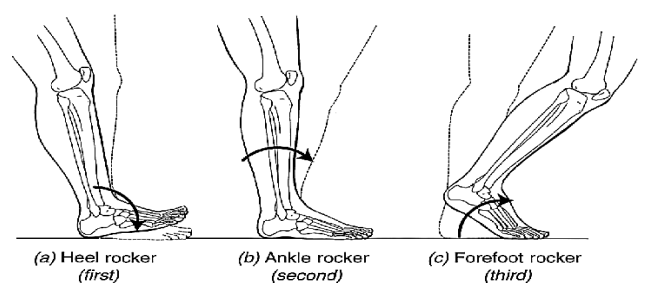

Fig. 5: The three foot rockers during stance phase.

$j$ th interval is a true stride we assume that the ankle speed at start $_{j}$ and $e n d_{j}$ is given by a lever arm:

$$
\left\{\begin{array}{cc}
V\left(\operatorname{start}_{j}\right)= & \Omega_{B^{t e r r}}\left(\operatorname{start}_{j}\right) \times\left(\begin{array}{l}
0 \\
0 \\
r
\end{array}\right), \\
V\left(\text { end }_{j}\right)= & \Omega_{B^{t e r r}}\left(\operatorname{end}_{j}\right) \times\left(\begin{array}{l}
0 \\
0 \\
r
\end{array}\right),
\end{array}\right.
$$

with $r$ the height relative to the ground of the device. In practice we set the value of $r$ at $8 \mathrm{~cm}$. From Equation 9 we can compute the forward speed $\left(V_{\text {start }_{j}, \text { end }_{j}}^{\text {for }}\right)$ and the backward speed $\left(V_{\text {start }_{j}, \text { end }_{j}}^{\text {back }}\right)$.

If the interval $j$ is a stride, these two speeds are close because we integrate the acceleration during a short period so that the drift stays small. In practice, $s_{t a r t}$ and $e n d_{j}$ do not necessarily correspond to the ankle rocker. In addition, taking $\mathrm{r}$ equals $8 \mathrm{~cm}$ is not realistic for all recordings. That is why we observe differences in the residuals $\mid V_{\text {start }_{j}, \text { end }_{j}}^{\text {for }}(t)-$ $V_{\text {start }_{j}, \text { end }_{j}}^{\text {back }}(t) \mid$ for $t$ in $\left[\right.$ start $_{j}, e$ end $\left._{j}\right]$. However, it can be much larger for movements that are not strides as Equation 15 do not stand. 
Then, thanks to Equation 11, we compute $V_{\text {start }_{j}, \text { end }_{j}}(t)$ on the studied interval. By integrating this pseudo-speed, we compute a pseudo-trajectory in the terrestrial reference frame $B^{\text {terr }}$, starting from the origin $(0,0,0)$ and ending in $\left(x_{e n d_{j}}, y_{e n d_{j}}, z_{\text {end }}\right)^{T}$ :

$$
\left(\begin{array}{l}
x_{\text {end }_{j}} \\
y_{\text {end }_{j}} \\
z_{\text {end }_{j}}
\end{array}\right)=\int_{\text {start }_{j}}^{\text {end }_{j}} V_{\text {start }_{j}, \text { end }_{j}}(u) d u .
$$

We consider a new terrestrial reference frame $B_{j}^{\text {terr }}$ with the $Z_{j}^{t e r r}$ axis still aligned with the gravity but with $X_{j}^{t e r r}$ defined by $\frac{\left(x_{e n d_{j}}, y_{e n d_{j}}, z_{e n d_{j}}\right)^{T}}{\left\|\left(x_{e n d_{j}}, y_{e n d_{j}}, z_{e n d_{j}}\right)^{T}\right\|}$. We note $R_{j}^{\text {terr }}$ the rotation matrix that projects the data from $B^{\text {terr }}$ to $B_{j}^{\text {terr }}$. For one stride interval $j$, we plot the trajectories in $B^{\text {terr }}$ and $B_{j}^{t e r r}$ (Figure $6)$. As we align the end of the trajectory with the $X_{j}^{\text {terr }}$ axis, the value on $Y_{j}^{t e r r}$ of the end point is null. The body frames

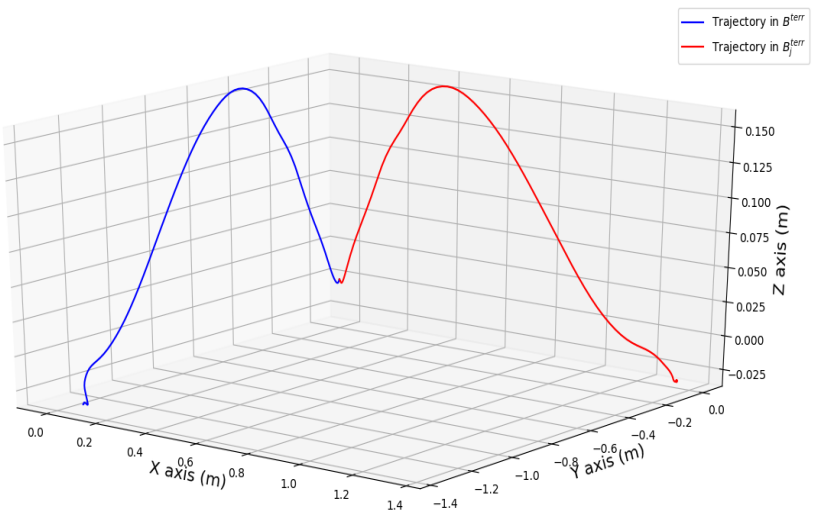

Fig. 6: Example of a computed pseudo-trajectory in $B^{\text {terr }}$ and $B_{j}^{t e r r}$.

$B_{j}^{t e r r}$ are not the same for all $j$ and for all recordings but they have the same building specifications. The 3-D interval data we have access to (trajectory, pseudo-speeds, residuals, acceleration and angular velocity) in $B_{j}^{t e r r}$ are independent to the initial position of the sensors. By proceeding this way We reduced drastically the complexity of the supervised learning problem. We compute features from signal processing techniques in time and frequency domains such as the mean, standard deviation, interquartile range, Fast Fourier Transform etc.

\section{Gradient Boosting Tree algorithm}

Following the strategy above, for each element of our database, 1657 features are computed. We want to build a binary classifier that decides if one interval is a stride. Several supervised statistical learning algorithms have been tested, notably random forests which are known to perform well in large dimensions, Support Vector Machine (SVM), LASSO regression and boosting algorithms such as Adaboost and GBT (Gradient Boosting Tree [20]). We evaluated their performance using the cross-validation method (10-fold cross-validation [21]). The chosen algorithm with the best results is GBT.
The general idea is to compute a series of (very weak) decision trees [22], learning at each step the prediction error of the previous aggregation. Let $\left\{\left(X_{1}, Y_{1}\right), \ldots,\left(X_{q}, Y_{q}\right), \ldots\left(X_{N}, Y_{N}\right)\right\}$ the elements of our database. As we computed 1657 features we have $X_{q}$ in $R^{1657}$. We note $Y_{q}$ the binary label of the interval $\left(Y_{q} \in\{0,1\}\right)$. We introduce $X=\left(X_{1}, \ldots, X_{N}\right)$ and $Y=\left(Y_{1}, \ldots, Y_{N}\right)$ for describing the GBT algorithm in the following.

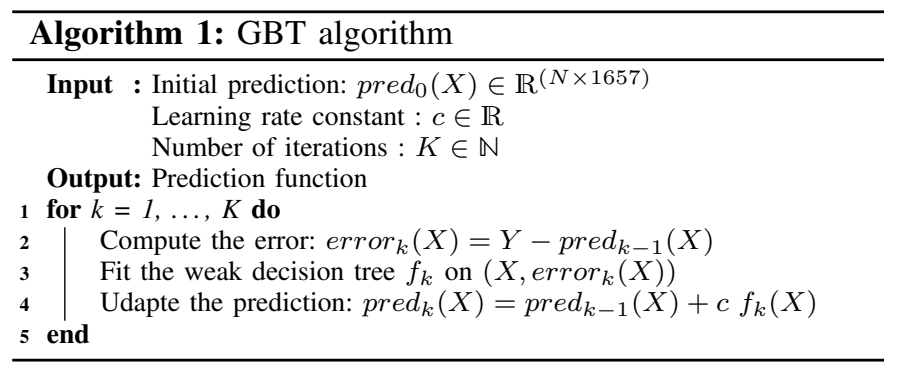

The parameters $c$ and $K$ have to be tuned to avoid the overfitting. They have been set by cross-validation.

\section{Overview}

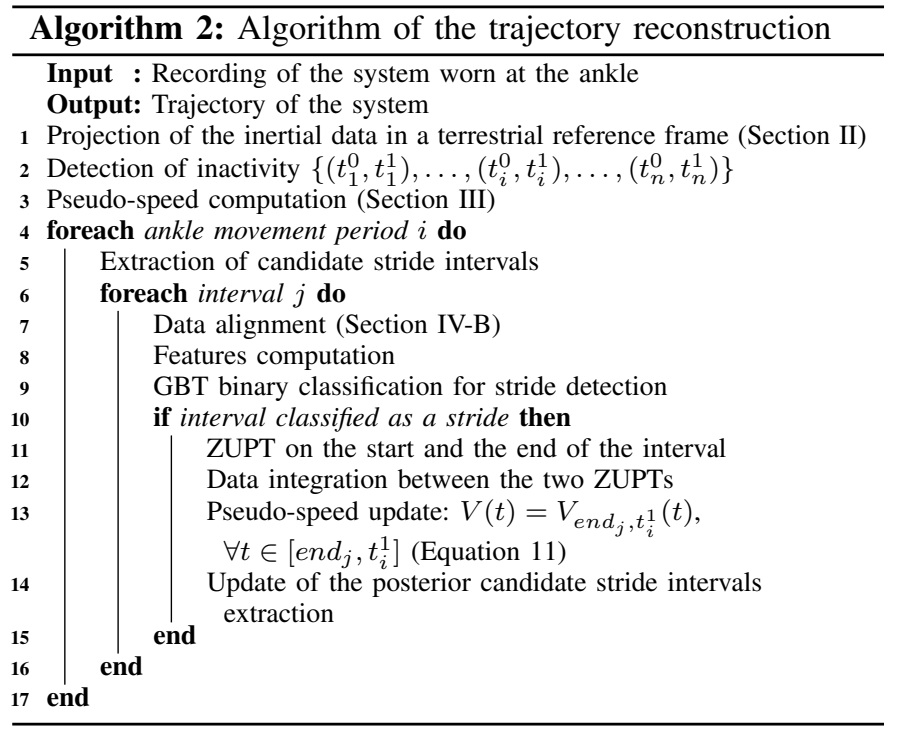

The step 13 of the Algorithm 2 is important to counter the integration drift if the ankle movement period $\left[t_{i}^{0}, t_{i}^{1}\right]$ is large. In that case, the weighted mean of forward speed and backward speed (Equations 10 and 11) may not overtake the integration errors for $t$ far from $t_{i}^{0}$ and $t_{i}^{1}$. With the pseudospeed update if strides are detected, the weighted mean is computed for smaller and smaller interval and overcomes the integration drift.

\section{APPLICATIONS}

The following section describes the performance of the stride detection and experimental results demonstrating the accuracy of the position estimation using the Algorithm 2. 


\section{A. Stride detector performance}

1) Performance on the database: The database contains 5779 intervals that do not correspond to strides (label 0) and 4870 stride intervals divided into different activities: "atypical step" that includes small step, side step, backward walking etc. walking, running, climbing and descending stairs. The label 0 intervals come from ankle movement that are not strides during bicycling for example and situations when the device is manipulated in the hand, carried in a pocket or backpack before being worn on the ankle. The cross-validation results using GBT are presented in the following confusion matrix (Table I).

\begin{tabular}{|l|c|c|}
\hline & Predicted 0 & Predicted 1 \\
\hline Actual 0 & 5659 & 120 \\
\hline Actual 1 & 191 & 4679 \\
\hline
\end{tabular}

TABLE I: Confusion matrix of GBT algorithm.

The mean error is less than $0.3 \%$. This score depends on the difficulty of the database. A lot of atypical strides and ankles movements (labelled -1) that look like true stride from inertial data point of view have been included in the database. As a result, the final score is slightly deteriorated but it leads to more robust classifier.

2) Performance in MOCAP sessions: That is why we also tested our algorithm on 7 MOCAP sessions. The stride intervals detected are manually validated with the video and the MOCAP altitude. The foot has to be on the ground to accept the detection. We ask the wearers to walk at three different paces, small steps and side steps (both sides). In order to observe left and right turns and straight lines, the wearers had to follow a loop path in both directions and an eight-shape reference trajectory. The results are presented in Table II and III.

\begin{tabular}{|c||c|c||c|c||c|c|}
\cline { 2 - 7 } \multicolumn{1}{c|}{} & \multicolumn{2}{c|}{ Slow walking } & \multicolumn{2}{c|}{ Medium walking } & \multicolumn{2}{c|}{ Fast walking } \\
\cline { 2 - 7 } \multicolumn{1}{c|}{} & Total & Detected & Total & Detected & Total & Detected \\
\hline Wearer 1 & 291 & $291-100 \%$ & 279 & $279-100 \%$ & 216 & $216-100 \%$ \\
\hline Wearer 2 & 306 & $306-100 \%$ & 261 & $261-100 \%$ & 195 & $195-100 \%$ \\
\hline Wearer 3 & 294 & $294-100 \%$ & 219 & $219-100 \%$ & 198 & $198-100 \%$ \\
\hline Wearer 4 & 297 & $297-100 \%$ & 267 & $267-100 \%$ & 228 & $228-100 \%$ \\
\hline Wearer 5 & 273 & $273-100 \%$ & 249 & $249-100 \%$ & 213 & $213-100 \%$ \\
\hline Wearer 6 & 345 & $345-100 \%$ & 339 & $339-100 \%$ & 327 & $327-100 \%$ \\
\hline Wearer 7 & 342 & $342-100 \%$ & 246 & $246-100 \%$ & 240 & $240-100 \%$ \\
\hline Total & 2148 & $2148-100 \%$ & 1860 & $1860-100 \%$ & 1617 & $1617-100 \%$ \\
\hline
\end{tabular}

TABLE II: Detection rate for walking phases.

All walking strides are detected. We can see on Figure 7 that the MOCAP database present diversified stride lengths and stride durations. It means our detection achieves $100 \%$ accuracy for walking phases with various paces. Some of the

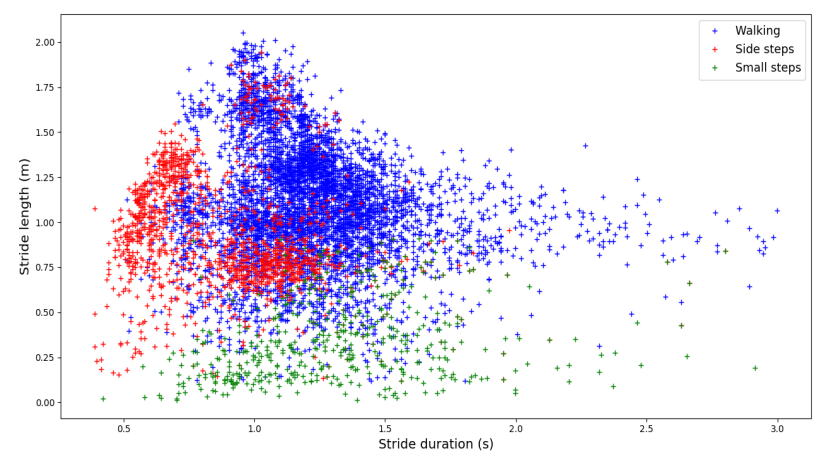

Fig. 7: Stride lengths as a function of stride durations. walking strides may appear very small but it is due to half turns of the MOCAP sessions. The foot comes in the end very close to the starting point of the stride.

\begin{tabular}{|c||c|c||c|c|}
\cline { 2 - 5 } \multicolumn{1}{c|}{} & \multicolumn{2}{c|}{ Small steps } & \multicolumn{2}{c|}{ Side steps } \\
\cline { 2 - 5 } \multicolumn{1}{c|}{} & Total & Detected & Total & Detected \\
\hline Wearer 1 & 88 & $88-100 \%$ & 287 & $285-99.3 \%$ \\
\hline Wearer 2 & 67 & $67-100 \%$ & 265 & $258-97.4 \%$ \\
\hline Wearer 3 & 107 & $102-95.3 \%$ & 143 & $138-96.5 \%$ \\
\hline Wearer 4 & 145 & $144-99.3 \%$ & 301 & $301-100 \%$ \\
\hline Wearer 5 & 65 & $62-92.3 \%$ & 246 & $244-99.2 \%$ \\
\hline Wearer 6 & 90 & $89-98.9 \%$ & 150 & $149-99.3 \%$ \\
\hline Wearer 7 & 48 & $44-91.7 \%$ & 200 & $199-99.5 \%$ \\
\hline Total & 610 & $596-97.7 \%$ & 1592 & $1574-98.9 \%$ \\
\hline
\end{tabular}

TABLE III: Detection rate for atypical strides.

Our algorithm does not detect all atypical strides but shows good results while most existing methods described in the literature do not detect them.

\section{B. Trajectory reconstruction performance}

1) Performance in MOCAP sessions: We first study the trajectory reconstruction performance by comparing the stride length computed by our algorithm to the MOCAP reference during sessions introduced in Section III-B. The results are presented in Tables IV and V.

\begin{tabular}{|c|c|c||c|c||c|c|}
\cline { 2 - 7 } \multicolumn{1}{c|}{} & \multicolumn{2}{c|}{ Slow walking } & \multicolumn{2}{c|}{ Medium walking } & \multicolumn{2}{c|}{ Fast walking } \\
\cline { 2 - 7 } \multicolumn{1}{c|}{} & Mean $(\mathrm{m})$ & Std $(\mathrm{m})$ & Mean $(\mathrm{m})$ & Std $(\mathrm{m})$ & Mean $(\mathrm{m})$ & Std $(\mathrm{m})$ \\
\hline Wearer 1 & 0.024 & 0.038 & 0.020 & 0.022 & 0.029 & 0.036 \\
\hline Wearer 2 & 0.026 & 0.039 & 0.016 & 0.018 & 0.025 & 0.034 \\
\hline Wearer 3 & 0.023 & 0.021 & 0.028 & 0.024 & 0.036 & 0.023 \\
\hline Wearer 4 & 0.028 & 0.020 & 0.028 & 0.021 & 0.023 & 0.022 \\
\hline Wearer 5 & 0.061 & 0.091 & 0.025 & 0.020 & 0.032 & 0.029 \\
\hline Wearer 6 & 0.018 & 0.016 & 0.024 & 0.024 & 0.043 & 0.039 \\
\hline Wearer 7 & 0.014 & 0.026 & 0.014 & 0.012 & 0.023 & 0.044 \\
\hline Total & 0.028 & 0.048 & 0.022 & 0.021 & 0.032 & 0.034 \\
\hline
\end{tabular}

TABLE IV: Absolute computed stride length error for walking phases.

\begin{tabular}{|c|c|c||c|c|}
\cline { 2 - 5 } \multicolumn{1}{c|}{} & \multicolumn{2}{c||}{ Small steps } & \multicolumn{2}{c|}{ Side steps } \\
\cline { 2 - 5 } \multicolumn{1}{c|}{} & Mean $(\mathrm{m})$ & Std $(\mathrm{m})$ & Mean $(\mathrm{m})$ & Std $(\mathrm{m})$ \\
\hline Wearer 1 & 0.027 & 0.070 & 0.044 & 0.106 \\
\hline Wearer 2 & 0.039 & 0.048 & 0.071 & 0.168 \\
\hline Wearer 3 & 0.057 & 0.082 & 0.070 & 0.177 \\
\hline Wearer 4 & 0.025 & 0.024 & 0.048 & 0.056 \\
\hline Wearer 5 & 0.049 & 0.082 & 0.022 & 0.046 \\
\hline Wearer 6 & 0.074 & 0.061 & 0.133 & 0.170 \\
\hline Wearer 7 & 0.039 & 0.055 & 0.053 & 0.116 \\
\hline Total & 0.048 & 0.069 & 0.056 & 0.129 \\
\hline
\end{tabular}

TABLE V: Absolute computed stride length error for atypical strides.

Our algorithm achieves similar performances than existing methods ( [23], [24]) for normal walking but also good performances for atypical strides (around $5 \mathrm{~cm}$ of absolute mean error) that are not even studied in the literature.

2) Performance in non controlled environment: In the following we validate the trajectory reconstruction in an everyday life situation. For this test an office worker has worn the system during 5 hours and 30 minutes. The aim is to test the step detector algorithm on strides performed naturally, including small steps. During those 5 hours and 30 minutes, the person was mostly sitting on his office chair. These periods are also interesting because the ankle does not remain inactive and it is important that no stride is wrongly detected. The recording 
contains 3 walking periods including up and down stairs in the first and last one.

The computed altitude of the first walking period is represented in Figure 8. From this graph we can detect when

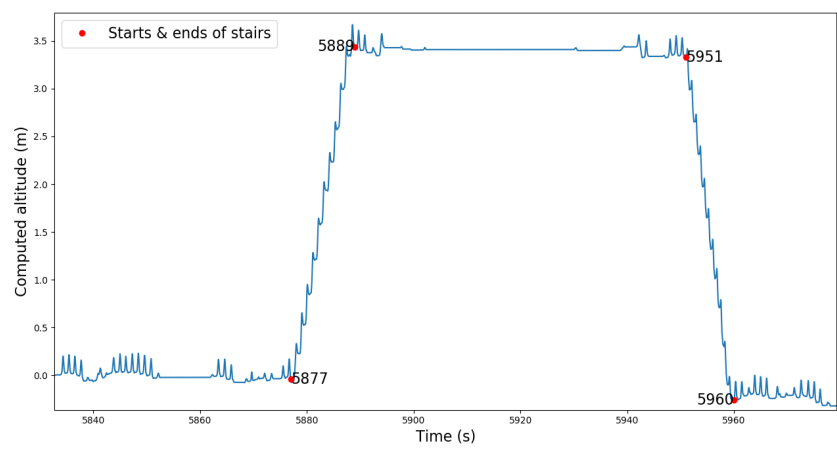

Fig. 8: Computed altitude during the first walking period.

the wearer is walking in the stairs. We plot in Figure 9 the computed trajectory in two dimensions on the plans of the ground floor and first floor depending on this altitude evolution. We add markers that indicate the beginning/end of the detected stairs from Figure 8 above. The colormap defines the time over the considered walking period: the more the greyscale is dark the more time has elapsed. In addition, the starting point is initialized with the coordinates $(0,0,0)$ and the computed trajectory has been rotated to have the correct initial direction.
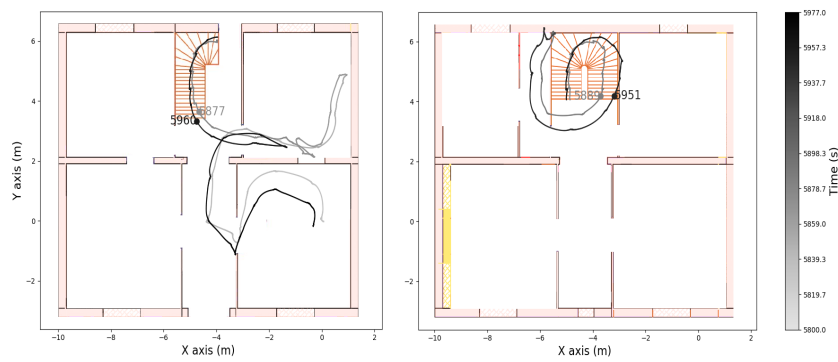

Fig. 9: Computed trajectory during the first walking period on the ground floor (left) and first floor (right).

During the second walking period, the wearer stays on the first floor. We plot in two dimensions the computed trajectory on the corresponding plan in Figure 10.

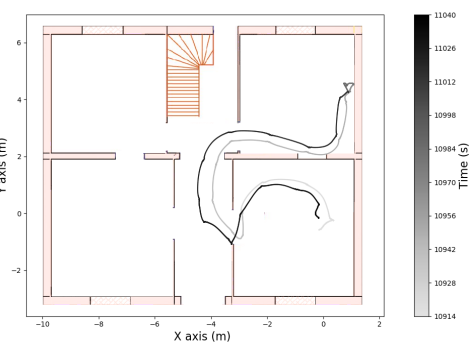

Fig. 10: Computed trajectory during the second walking period on the ground floor.

The computed altitude of the third walking period is represented in Figure 11. Using the same first walking period

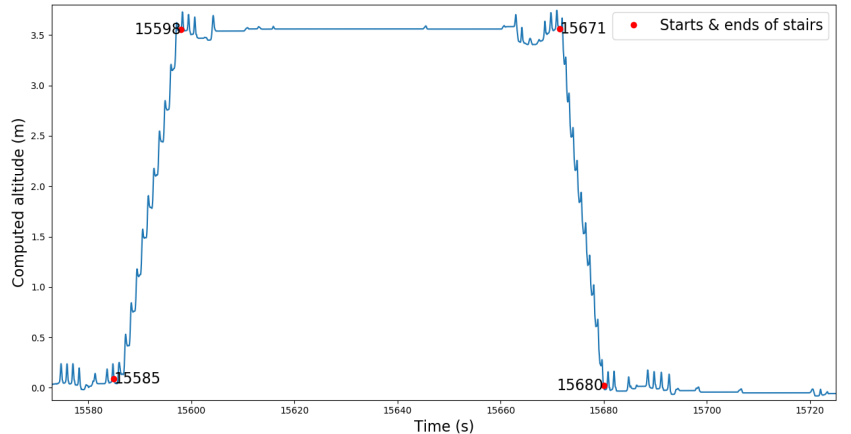

Fig. 11: Computed altitude during the third walking period.

approach, we plot in Figure 12 the computed trajectory in two dimensions and we add markers that indicate the beginning/end of the detected stairs from Figure 11 above. This
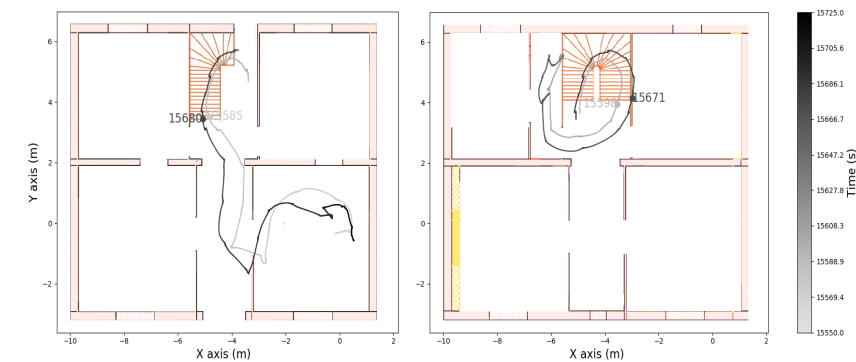

Fig. 12: Computed trajectory during the third walking period on the ground floor (left) and the first floor (right).

experiment illustrates the good performance of the trajectory reconstruction in a difficult environment with narrow ways, small rooms and corridors. In this context, the computed trajectory almost never crosses the walls and we can identify in what room the wearer is at any time or when he is taking the stairs. Figure 8 and Figure 11 indicate a difference in the computed altitude of 3.4 meters for both stairs phases that are composed of 21 stair-treads of $15.4 \mathrm{~cm}$ height. The true altitude of the first ground is 3.234 meters so the altitude mean error is less than $1 \mathrm{~cm}$ for each stair-tread. In addition, the starting point of the second and third walking period correspond to the ending point of the previous one. It means that no stride is wrongly detected when the wearer is on his chair and moving his ankle.

\section{CONCLUSION}

This paper describes an algorithm that compute the trajectory of a pedestrian wearing an ankle worn inertial device. This work is divided in four main steps:

- The projection of the inertial data in a terrestrial reference frame by detecting the gravity in the accelerations.

- The extraction of candidate intervals based on the computed pseudo-speed that may correspond to strides.

- The binary classification of the intervals using the Gradient Boosting Tree algorithm. 
- The computation of an inspired ZUPT technique for the detected stride.

For normal walking it shows good results achievable with existing algorithms with $100 \%$ stride detection rate and around $3 \mathrm{~cm}$ of absolute mean error for the stride length. In addition, the method described in this paper also has a good sensitivity for atypical strides such as small steps, side steps contrary to most algorithms proposed in the literature. It achieves more than $98 \%$ detection rate and the absolute mean error of the stride length is about $5 \mathrm{~cm}$.

Moreover existing approaches are likely to produce detection error when the system wearer is moving his ankle but not walking (e.g. sitting). This is a problem as non walking motion would be integrated erroneously in the trajectory. Our algorithm handles those situations without false detection. A challenging test have been performed by recording an office worker during 5 hours and 30 minutes. The building presented narrow areas and stairs forcing the wearer to perform atypical steps. He stayed also for several hours sitting on his chair keeping his ankle moving. In this context, the computed trajectory illustrates the good performance by barely never crossing the walls of the building map and no stride is wrongly detected as false positive.

\section{ACKNOWLEDGMENT}

This work was supported by the French Délégation Générale de l'Armement (DGA) and by ANR project TopData ANR17-MALN-0003.

\section{REFERENCES}

[1] Y. Chen and H. Kobayashi, "Signal strength based indoor geolocation," Proceedings of the IEEE International Conference on Communications, pp. 436-439, 2002.

[2] V. Renaudin, "Uwb and mems based indoor navigation," The Journal of Navigation, vol. 61, pp. 369-384, 2008.

[3] S. S. et al., "Hybrid localization using uwb and inertial sensors," IEEE Int. Conf. Ultra-Wideband (ICUWB), pp. 89-92, 2008.

[4] E. Dorveaux, "Magneto-inertial navigation: principles and application to an indoor pedometer," PhD thesis, École Nationale Supeure des Mines de Paris, 2011.

[5] C. I. Chesneau, M. Hillion, and C. Prieur, "Motion estimation of a rigid body with an ekf using magneto-inertial measurements," Indoor Positioning and Indoor Navigation (IPIN), 2016.

[6] K. Abdulrahim, T. Moore, C. Hide, and C. Hill, "Understanding the performance of zero velocity updates in mems-based pedestrian navigation," International Journal of Advancements in Technology, vol. 5, no. $2,2014$.

[7] E. Foxlin, "Pedestrian tracking with shoe-mounted inertial sensors," IEEE Computer graphics and applications, pp. 38-46, 2005.

[8] A. M. Sabatini, "Quaternion-based strap-down integration method for applications of inertial sensing to gait analysis," Medical and Biological Engineering and Computing, pp. 94-101, 2005.

[9] S. J. Bamberg, A. Y. Benbasat, D. M. Scarborough, E. E. Krebs, and J. A. Paradiso, "Gait analysis using a shoe-integrated wireless sensor system," IEEE transactions on information technology in biomedicine, pp. 413-423, 2008.

[10] M. Susi, V. Renaudin, and G. Lachapelle, "Motion mode recognition and step detection algorithms for mobile phone users," Sensors, pp. 15391562, 2013.

[11] B. Florentino-Liano, N. O'Mahony, and A. Artes-Rodriguez, "Human activity recognition using inertial sensors with invariance to sensor orientation," Conf. Cognitive Information Processing (CIP), 2012.

[12] S. Ghose, J. Mitra, M. Karunanithi, and J. Dowling, "Human activity recognition from smart-phone sensor data using a multi-class ensemble learning in home monitoring," Stud Health Tehcnol Inform, 2015.
[13] N. Castaneda and S. Lamy-Perbal, "An improved shoe-mounted inertial navigation system," International conference on indoor positioning and indoor navigation (IPIN), 2010.

[14] J.-L. Carrera, Z. Zhao, T. Braun, and Z. Li, "A real-time indoor tracking system by fusing inertial sensor, radio signal and floor plan," International conference on indoor positioning and indoor navigation (IPIN), 2016.

[15] A. Norrdine, Z. Kasmi, and J. Blankenbach, "Step detection for zuptaided inertial pedestrian navigation system using foot-mounted permanent magnet," International conference on indoor positioning and indoor navigation (IPIN), 2016.

[16] T. Moder, K. W. nd P. Hafner, and M. Wieser, "Smartphone-based indoor positioning utilizing motion recognitiont," International conference on indoor positioning and indoor navigation (IPIN), 2015.

[17] S. Y. Cho and C.-G. Park, "Mems based pedestrian navigation system," Journal of Navigation, vol. 59, pp. 135-153, 2006.

[18] B. Beaufils, F. Chazal, M. Grelet, and B. Michel, "Stride detection for pedestrian trajectory reconstruction: a machine learning approach based on geometric patterns," International conference on indoor positioning and indoor navigation (IPIN), 2017.

[19] Z. O. Abu-Faraj, G. F. H. andPeter A Smith, and S. Hassani, "Human gait and clinical movement analysis," Wiley Encyclopedia of Electrical and Electronics Engineering, 2015.

[20] J. H. Friedman, "Computational statistics and data analysis," 2002.

[21] M. Stone, "Cross-validatory choice and assessment of statistical predictions," Journal of the Royal Statistical Society, pp. 111-147, 1974.

[22] L. Breiman, J. Friedman, R. Olshen, and C. Stone, "Classification and regression tree," 1984.

[23] N.-H. Ho, P. H. Truong, and G.-M. Jeong, "Step-detection and adaptive step-length estimation for pedestrian dead-reckoning at various walking speeds using a smartphone," Sensors, 2016.

[24] J. Hannink, C. F. P. Thomas Kautz, J. Barth, S. Schlein, K.-G. G. mann, J. Klucken, and B. M. Eskofier, "Stride length estimation with deep learning," IEEE EMBS, 2017. 